\title{
MULTIPLE SOLUTIONS OF NONLINEAR BOUNDARY VALUE PROBLEMS WITH OSCILLATORY SOLUTIONS
}

\author{
S. OGORODNIKOVA and F. SADYRBAEV
}

Daugavpils University

Parades str.1, Daugavpils LV-5400, Latvia

E-mail: oglana@tvnet.lv; felix@dau.lv

Received October 15, 2005; revised June 01, 2006; published online December 15, 2006

\begin{abstract}
We consider two second order autonomous differential equations with critical points, which allow the detection of an exact number of solutions to the Dirichlet boundary value problem. Non-autonomous equations with similar behaviour of solutions also are considered. Estimations from below of the number of solutions to the Dirichlet boundary value problem are given.

Key words: singular points, multiple solutions, heteroclinic solutions, homoclinic solutions
\end{abstract}

\section{Introduction}

Main issues in the theory of boundary value problems for ordinary differential equations are the existence and uniqueness of solutions. A lot of practically important problems possess however multiple solutions. There is intensive literature devoted to multiplicity of solutions. Let us mention the works $[1,6,7]$ and references therein.

In our research we are motivated by the method proposed by A.I. Perov $[4,5]$ (the respective results can be found also in the book [2, Ch. 15]). His method results in estimation of the number of solutions of the boundary value problem

$$
\left\{\begin{array}{l}
x^{\prime}=h(t, x, y), \\
y^{\prime}=f(t, x, y), \\
a_{1} x(a)-b_{1} x^{\prime}(a)=0, \\
a_{2} x(b)-b_{2} x^{\prime}(b)=0 .
\end{array}\right.
$$

These estimations are based on comparison of the behaviour of solutions in some neighbourhood of the zero solution and at infinity. Notice that the zero 
solution exists since $h(t, 0,0)=f(t, 0,0)=0$. It is convenient to explain the result of Perov in terms of the angular function $\varphi(t)$, which can be introduced by the following relations

$$
x=\rho \sin \varphi, \quad y=\rho \cos \varphi, \quad \rho^{2}=x^{2}+y^{2} .
$$

We get the following equations for the functions $\varphi$ and $\rho$ :

$$
\left\{\begin{array}{l}
\varphi^{\prime}=\frac{1}{\rho}(h \cos \varphi-f \sin \varphi) \\
\rho^{\prime}=h \sin \varphi+f \cos \varphi
\end{array}\right.
$$

In the polar coordinates the first boundary condition may be written as

$$
\varphi(a)=\alpha:= \begin{cases}\arctan \frac{b_{1}}{a_{1}}, & \text { if } a_{1} \neq 0, \\ \frac{\pi}{2}, & \text { if } a_{1}=0 .\end{cases}
$$

The second boundary condition takes the form

$$
\varphi(b)=\beta(\bmod \pi), \quad \text { where } \beta= \begin{cases}\arctan \frac{b_{2}}{a_{2}}, & \text { if } a_{2} \neq 0, \\ \frac{\pi}{2}, & \text { if } a_{2}=0 .\end{cases}
$$

Set

$$
\rho_{0}= \pm \sqrt{x^{2}(a)+y^{2}(a)}
$$

Suppose that a solution $\varphi\left(t ; \rho_{0}\right)$ of the system (1.2), which is defined by the initial condition $\varphi(a)=\alpha$ for small $\rho_{0}>0$, takes exactly $m$ values of the form $\beta(\bmod \pi)$. Moreover, assume that a solution $\varphi\left(t ; \rho_{0}\right)$, which is defined by the initial condition $\varphi(a)=\alpha$ for large values of $\rho_{0}>0$, takes $n$ values of the form $\beta(\bmod \pi)$. If $n$ is, say, greater than $m$, then there exist at least $n-m$ values of $\rho_{0}$, which give rise to solutions of the BVP. Another at least $n-m$ solutions are obtained for $\rho_{0} \in(-\infty, 0)$. Then there exist at least $2|n-m|$ nontrivial solutions of the problem.

Figure 1 visualizes the case of $\alpha=0, \beta=\frac{\pi}{2}$ (the Dirichlet boundary conditions $x(a)=0, x(b)=0)$ and $n=0, m=1$. Two possible solutions of the BVP are represented by two semicircles. Due to different rates of whirling of solutions near the zero and at infinity multiple solutions of the problem appear.

The above mentioned result by Perov is more general than that described by Fig. 1, since equations in (1.1) are non-autonomous.

Our aim in this paper is the following. We consider the second order equations, which are equivalent to two-dimensional systems, which are similar to those treated by Perov and which, moreover, can have hetero- and homoclinic type solutions. First, we consider autonomous equations which have singular points of the type saddle-center-saddle. This equation has a heteroclinic solution and it may have multiple solutions of the Dirichlet problem. 


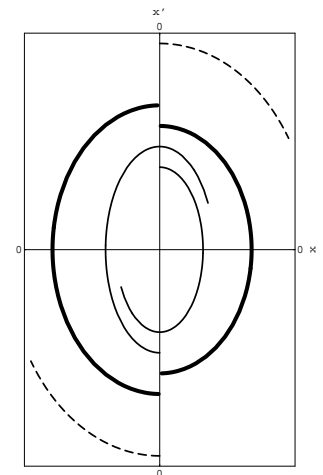

Figure 1. Perov's result ( $m=1, n=0)$, bold-orbits of solutions to BVP; normalorbits at zero; dashed-orbits at infinity.

Then, these results are generalized to the case of non-autonomous equation, which has a solution, defined on a finite interval and which possesses some properties of a heteroclinic solution. Similar situation is considered for autonomous equations which have singular points in combination center-saddle. This equation has a homoclinic solution and it may also have multiple solutions of the Dirichlet problem.

\section{Autonomous Equations, I}

Consider the problem

$$
\left\{\begin{array}{l}
x^{\prime \prime}=-\alpha x+x^{3}, \\
x(0)=0, \quad x(1)=0,
\end{array}\right.
$$

where $\alpha$ is a positive parameter. Equation (2.1) describes, for instance, the stationary solutions of the Fisher - Kolmogorov equation:

$$
\frac{\partial u}{\partial t}=\frac{d^{2} u}{d t^{2}}+\alpha u-u^{3}
$$

Many examples of this kind can be found in the book [3].

The equivalent system

$$
\left\{\begin{array}{l}
x^{\prime}=y, \\
y^{\prime}=-\alpha x+x^{3}
\end{array}\right.
$$

has a center at $(0 ; 0)$ and two saddle points at $(-\sqrt{\alpha} ; 0)$ and $(\sqrt{\alpha} ; 0)$. The heteroclinic orbit connects two saddle points. The respective heteroclinic solution has "an infinite" period [8]. 


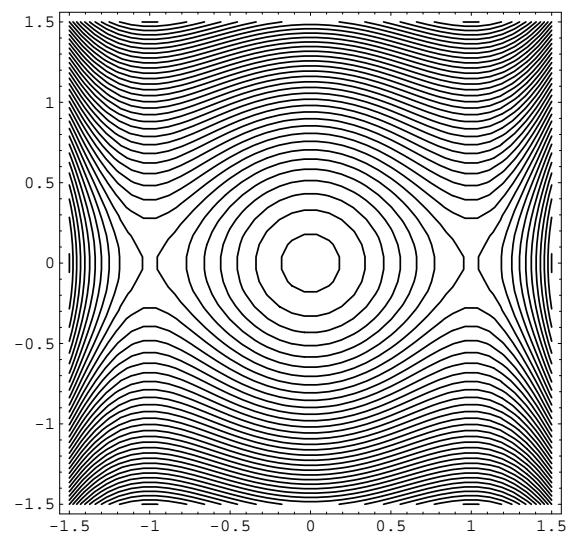

Figure 2. Phase portrait of solutions of equation $x^{\prime \prime}=-x+x^{3}$.

Proposition 1. Let the condition

$$
\pi^{2} n^{2}<\alpha<\pi^{2}(n+1)^{2}
$$

hold, where $n$ is a non-negative integer. Then problem (2.1) has exactly $2 n$ nontrivial solutions.

Proof. Consider solutions $x(t ; \gamma)$ of equation (2.1), which satisfy the initial conditions

$$
x(0)=0, \quad x^{\prime}(0)=\gamma .
$$

The phase portrait for the system (2.2) is well known (see [8], for example) and is depicted in Fig.2. Consider the region on a phase plane bounded by two heteroclinic solutions. Any phase orbit in this region satisfies the relation

$$
x^{2}+\alpha x^{2}=\frac{1}{2} x^{4}+\gamma^{2},
$$

or

$$
x^{\prime 2}+\alpha x^{2}=\frac{1}{2} x^{4}+\alpha A^{2}-\frac{1}{2} A^{4},
$$

where $A$ stands for the maximal value of the respective solution $x(t ; \gamma)$. Therefore $\gamma^{2}=\alpha A^{2}-\frac{1}{2} A^{4}$. Notice that the maximum of a heteroclinic solution (which is attained at $t=+\infty$ ) is $A=\sqrt{\alpha}$. Thus the respective value $x^{\prime}(0)=\gamma_{0}$ is equal to $\frac{\sqrt{2}}{2} \alpha$.

Let $\gamma \in\left(0, \gamma_{0}\right)$. Then any solution $x(t ; \gamma)$ is periodic. We claim that the period $T(\gamma)$ is a monotonically increasing function of $\gamma$. Indeed, one has from (2.6) that

$$
\frac{d x}{d t}=\sqrt{-\alpha x^{2}+\frac{1}{2} x^{4}+\alpha A^{2}-\frac{1}{2} A^{4}}
$$

in the interval $\left[0, T_{A}\right]$, where $T_{A}$ stands for a quarter of the period of a solution $x(t ; \gamma)$. One has that 


$$
\begin{aligned}
T_{A} & =\int_{0}^{A} \frac{d x}{\sqrt{\left(\alpha A^{2}-\frac{1}{2} A^{4}\right)-\left(\alpha x^{2}-\frac{1}{2} x^{4}\right)}} \\
\left(\xi=\frac{x}{A}\right) & =\int_{0}^{1} \frac{d \xi}{\sqrt{\left(\alpha-\frac{1}{2} A^{2}\right)-\left(\alpha \xi^{2}-\frac{1}{2} \xi^{4} A^{2}\right)}} \\
& =\int_{0}^{1} \frac{d \xi}{\sqrt{\alpha\left(1-\xi^{2}\right)-\frac{1}{2} A^{2}\left(1-\xi^{4}\right) .}}
\end{aligned}
$$

It is clear that $T_{A}$ increases as $A$ increases. Therefore $T_{A}(\gamma)$ monotonically increases as a function of $\gamma$. Since the half-period of an upper $\left(x^{\prime} \geq 0\right)$ heteroclinic solution is infinite and orbits are symmetric with respect to both $x$ and $x^{\prime}$ axes it is true that $\lim _{\gamma \rightarrow \gamma_{0}} T_{A}(\gamma)=+\infty$.

On the other hand, $T_{A}$ for small $\gamma>0$ is defined by a quarter-period of a solution of the respective equation of variations (with respect to the trivial solution of (2.1))

$$
y^{\prime \prime}=-\alpha y, \quad y(0)=0, y^{\prime}(0)=1 .
$$

Since $y(t)=\frac{1}{\sqrt{\alpha}} \sin (\sqrt{\alpha} t)$ one gets that $T_{A}(\gamma) \rightarrow \frac{\pi}{2 \sqrt{\alpha}}$ as $\gamma \rightarrow 0$.

Due to the conditions (2.3) the function $y(t)$ has exactly $n$ zeros in the interval $(0 ; 1)$ and does not vanish at $t=1$. The same is true for solutions of the problem (2.1), (2.4) for small enough $\gamma>0$.

The zeros of solutions $x(t ; \gamma)$ monotonically increase and leave the interval $(0 ; 1]$ passing through the right end point as $\gamma \rightarrow \gamma_{0}$. If for some $\gamma$ a solution $x(t ; \gamma)$ has a zero at $t=1$, then $x(t ; \gamma)$ is a solution to the boundary value problem (2.1). Thus there exist exactly $n$ nontrivial solutions of the problem for $\gamma \in\left(0, \gamma_{0}\right)$.

Similarly $n$ solutions to the boundary value problem appear for $\gamma \in$ $\left(-\gamma_{0}, 0\right)$. Solutions $x(t, \gamma)$ of the initial value problem $(2.1),(2.4)$ do not vanish for $|\gamma|>\left|\gamma_{0}\right|$.

So far we have considered two regions on a phase plane: one is a region $H$ bounded by two symmetric heteroclinic orbits and the exterior region $H_{\text {ext }}=$ $R \backslash H$. There are exactly $2 n$ nontrivial solutions of the problem (2.1) in $H$. The $H_{\text {ext }}$ does not contain solutions of the boundary value problem. Therefore problem (2.1) has exactly $2 n$ nontrivial solutions (the total number of solutions is $2 n+1$, when the trivial solution included).

\section{Non-Autonomous Equations, I}

Consider the problem

$$
\left\{\begin{array}{l}
x^{\prime \prime}=f(t, x), \\
x(0)=x(1)=0,
\end{array}\right.
$$

where function $f$ satisfies the the following conditions: 
(A1) $f$ and $f_{x}$ are $C(I \times R)$-functions;

(A2) $f(t, 0) \equiv 0$;

(A3) $x f(t, x)>0$ for $t \in I,|x|>M$, where $M>0$ is a constant;

(A4) there exists a solution $\eta(t)$ of the problem $(3.1), \eta(0)=0, \eta^{\prime}(0)>0$ such that $\eta(t)$ does not vanish in the interval $(0 ; 1]$;

(A5) there exists a solution $\xi(t)$ of the problem $(3.1), \xi(0)=0, \xi^{\prime}(0)<0$ such that $\xi(t)$ does not vanish in the interval $(0 ; 1]$;

(A6) solutions of equation (3.1) extend to the interval $(0 ; 1]$.

Theorem 1. Let conditions $(A 1)-(A 6)$ hold. Assume also that a solution $y(t)$ of the Cauchy problem

$$
\left\{\begin{array}{l}
y^{\prime \prime}=f_{x}(t, 0) y \\
y(0)=0, \quad y^{\prime}(0)=1
\end{array}\right.
$$

has exactly $n$ zeros $(n=0,1, \ldots)$ in the interval $(0,1)$ and $y(1) \neq 0$. Then problem (3.1) has at least $2 n$ nontrivial solutions.

Proof. Consider solutions $x(t ; \gamma)$ of equation (3.1), which satisfy the initial conditions (2.4). Let us introduce the polar coordinates by

$$
x=\rho \sin \varphi, \quad x^{\prime}=\rho \cos \varphi .
$$

Notice that $\rho(t)$ cannot vanish for nontrivial solutions since $x \equiv 0$ is a solution. Equation (3.1) in polar coordinates takes the form

$$
\left\{\begin{array}{l}
\varphi^{\prime}=\cos ^{2} \varphi-\frac{1}{\rho} f(t, \rho \sin \varphi) \sin \varphi \\
\rho^{\prime}=\rho \sin \varphi \cos \varphi+f(t, \rho \sin \varphi) \cos \varphi
\end{array}\right.
$$

Consider functions $\varphi(t ; \gamma)$ and $\rho(t ; \gamma)$ which satisfy the initial conditions

$$
\varphi(0 ; \gamma)=0, \quad \rho(0 ; \gamma)=|\gamma| .
$$

We write linear equation of variations (3.2) in polar coordinates

$$
y=r \sin \Theta, \quad y^{\prime}=r \cos \Theta
$$

it takes the following form

$$
\left\{\begin{array}{l}
\Theta^{\prime}=\cos ^{2} \Theta-f_{x}(t, 0) \sin ^{2} \Theta, \\
r^{\prime}=r \sin \Theta \cos \Theta+f_{x}(t, 0) r \sin \Theta \cos \Theta .
\end{array}\right.
$$

Due to conditions (A1), (A2) and (A6) the results of $[2$, Ch. $3, \S 15]$ are applicable. Since a solution $y(t)$ of problem $(3.2)$ has exactly $n$ zeros in $(0,1)$ and $y(1) \neq 0$ one has that $\pi n<\Theta(1)<\pi(n+1)$. It follows from the proof of 
Theorem 15.11 in [2] that the same inequalities hold for the angular function $\varphi$, i.e.

$$
\pi n<\varphi(1 ; \gamma)<\pi(n+1),
$$

if $\gamma>0$ is small enough. On the other hand, by condition (A4) we have

$$
0<\varphi\left(1 ; \gamma_{\eta}\right)<\pi,
$$

where $\gamma_{\eta}:=\eta^{\prime}(0)$. Taking into account the continuous dependence of solutions of the equation on initial data and since there exists a trivial solution $x \equiv 0$ we get that function $\varphi(1 ; \gamma)$ is continuous. Comparison of $(3.5)$ and (3.6) gives that there exist at least $n$ solutions for $\gamma \in\left(0, \gamma_{\eta}\right)$.

Another $n$ solutions are obtained by considering $x(t ; \gamma)$ for $\gamma \in\left(\gamma_{\xi}, 0\right)$. Thus there exist at least $2 n$ nontrivial solutions of the problem.

\section{Autonomous Equations, II}

Consider the problem

$$
\left\{\begin{array}{l}
x^{\prime \prime}=-\alpha x+x^{2}, \\
x(0)=0, \quad x(1)=0,
\end{array}\right.
$$

where $\alpha$ is a positive parameter.

The equivalent system

$$
\left\{\begin{array}{l}
x^{\prime}=y, \\
y^{\prime}=-\alpha x+x^{2}
\end{array}\right.
$$

has a center at $(0 ; 0)$ and a saddle point at $(\alpha ; 0)$ as depicted in Fig. 3. The homoclinic orbit connects the saddle point to itself. It has "an infinite" period [8].

Consider equation (4.1) with the following initial conditions

$$
x(0)=0, \quad x^{\prime}(0)=\gamma .
$$

Denote the respective solution by $x(t ; \gamma)$. The region bounded by the homoclinic solution is filled by closed orbits which can be parameterized by $\gamma$. Let $\gamma_{0}=x_{\text {homoclinic }}^{\prime}(0)>0$, where the homoclinic solution is such that $x(0)=0$. Let $A$ and $-m$ stand for respectively maximum and minimum of $x(t ; \gamma), \gamma \in\left(0, \gamma_{0}\right)$. For all such solutions $x(t ; \gamma)$ the relations

$$
\begin{aligned}
x^{\prime 2}+\alpha x^{2} & =\frac{2}{3} x^{3}+\gamma^{2}, \\
x^{\prime 2}+\alpha x^{2} & =\frac{2}{3} x^{3}+\alpha A^{2}-\frac{2}{3} A^{3}, \\
x^{2}+\alpha x^{2} & =\frac{2}{3} x^{3}+\alpha m^{2}+\frac{2}{3} m^{3}
\end{aligned}
$$




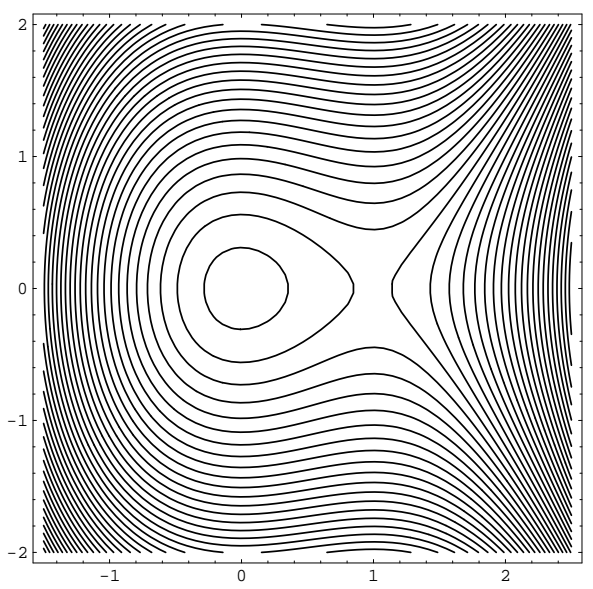

Figure 3. Phase portrait of solutions of equation $x^{\prime \prime}=-x+x^{2}$.

are satisfied for any $t>0$. Let $T_{1}, T_{2}, T_{3}, T_{4}$ be respectively the times needed for a point to move along the closed orbit from the point $(0, \gamma)$ to $(A, 0)$, from $(A, 0)$ to $(0,-\gamma)$, from $(0,-\gamma)$ to $(-m, 0)$ and from $(-m, 0)$ to $(0, \gamma)$. It follows that $T_{1}=T_{2}$ and $T_{3}=T_{4}$ since any orbit is symmetric with respect to the $x$-axis. The entire period of a solution $x(t ; \gamma)$ is the sum $T_{1}+T_{2}+T_{3}+T_{4}$ or $2 T_{1}+2 T_{3}$.

Lemma 1. $T_{1}$ is given by

$$
T_{1}=\int_{0}^{1} \frac{d \xi}{\sqrt{\alpha\left(1-\xi^{2}\right)-\frac{2}{3} A\left(1-\xi^{3}\right)}}
$$

and it is an increasing function of $\gamma \in\left(0, \gamma_{0}\right)$. Moreover, $T_{1} \rightarrow+\infty$ as $\gamma \rightarrow \gamma_{0}$.

Proof. Let us integrate (4.4) in the interval $\left(0, T_{1}\right)$. Then

$$
\begin{gathered}
T_{1}=\int_{0}^{A} \frac{d x}{\sqrt{\alpha A^{2}-\frac{2}{3} A^{3}-\alpha x^{2}+\frac{2}{3} x^{3}}} \\
\left(\xi=\frac{x}{A}\right)=\int_{0}^{1} \frac{d \xi}{\sqrt{\alpha\left(1-\xi^{2}\right)-\frac{2}{3} A\left(1-\xi^{3}\right)}} .
\end{gathered}
$$

Evidently, $T_{1}$ increases as $A$ increases. It follows from (4.3) and (4.4) that

$$
\gamma^{2}=\alpha A^{2}-\frac{2}{3} A^{3}
$$

Therefore $\gamma(A)$ is an increasing function for $A \in(0, \alpha)$. Thus $T_{1}(\gamma)$ is an increasing function in the interval $\left(0, \gamma_{0}\right)$ and the homoclinic solution has an infinite period $T_{1}(\gamma) \rightarrow+\infty$ as $\gamma \rightarrow \gamma_{0}$. 
Lemma 2. Suppose that $x^{\prime}(0)=\gamma \in(0,+\infty)$. Let $T_{4}$ be the time needed for a point to move along the trajectory $\left(x(t ; \gamma), x^{\prime}(t ; \gamma)\right)$ from the point $(-m, 0)$ to the point $(0, \gamma)$, here $m>0$. The value of $T_{4}$ is given by

$$
T_{4}=\int_{0}^{1} \frac{d \xi}{\sqrt{\alpha\left(1-\xi^{2}\right)+\frac{2}{3} m\left(1-\xi^{3}\right)}}
$$

and it is a decreasing function of $\gamma$. Moreover, $T_{4} \rightarrow 0$ as $\gamma \rightarrow+\infty$.

Proof. Let us notice that for trajectories which are located in a half-plane $x \leq 0$ the formulas (4.3) and (4.5) are valid. Consider a solution $x(t)$ of equation (4.1) which satisfies the initial conditions $x(0)=-m, x^{\prime}(0)=0$. One gets by integrating (4.5) that

$$
\begin{aligned}
T_{4} & =\int_{-m}^{0} \frac{d x}{\sqrt{\alpha m^{2}+\frac{2}{3} m^{3}-\alpha x^{2}+\frac{2}{3} x^{3}}} \\
(z=-x) & =\int_{0}^{m} \frac{d z}{\sqrt{\alpha m^{2}+\frac{2}{3} m^{3}-\alpha z^{2}-\frac{2}{3} z^{3}}} \\
\left(\xi=\frac{z}{m}\right) & =\int_{0}^{1} \frac{d \xi}{\sqrt{\alpha+\frac{2}{3} m-\frac{2}{3} \xi^{2} m \xi}} \\
& =\int_{0}^{1} \frac{d \xi}{\sqrt{\alpha\left(1-\xi^{2}\right)+\frac{2}{3} m\left(1-\xi^{3}\right)}} .
\end{aligned}
$$

Therefore $T_{4}$ monotonically decreases as a function of $m$. Since $m$ and $\gamma$ due to (4.3) and (4.5) relate as

$$
\gamma^{2}=\alpha m^{2}+\frac{2}{3} m^{3}
$$

and $m \rightarrow+\infty$ as $\gamma \rightarrow+\infty$ we get the proof.

Corollary 1 . The time $T_{-}$, needed for a point $(-\gamma, 0)$ to move to a position $(0, \gamma)$ along the orbit on a phase plane, decreases as $\gamma$ increases and $T_{-}(\gamma) \rightarrow 0$ as $\gamma \rightarrow+\infty$.

Proof. The proof follows from Lemma 2 and observations that $T_{-}=T_{3}+T_{4}$ and $T_{3}=T_{4}$.

Proposition 2. Suppose that the following condition

$$
\pi^{2} n^{2}<\alpha<\pi^{2}(n+1)^{2}
$$

holds, where $n$ is a positive integer. Then problem (4.1) has at least $(2 n-1)$ nontrivial solutions. 
Proof. Consider the initial value problem (4.1). If the parameter $\gamma>0$ is small enough, then solutions $x(t ; \gamma)$ have exactly $n$ zeros. If $\gamma=\gamma_{0}$ then $x\left(t ; \gamma_{0}\right)$ does not vanish in the interval $(0 ; 1]$. Hence there exist at least $n$ solutions of problem (4.1).

If the parameter $\gamma<0$ is small enough then solutions $x(t ; \gamma)$ have exactly $n$ zeros. If $\gamma$ tends to $-\infty$ then by Lemma 2 solutions $x\left(t ; \gamma_{0}\right)$ have exactly 1 zero in the interval $(0 ; 1)$. Hence there exist at least $(n-1)$ solutions of problem (4.1) for $\gamma<0$. Totally we get at least $(2 n-1)$ solutions of the given problem.

\section{Non-Autonomous Equations, II}

Consider the problem

$$
\left\{\begin{array}{l}
x^{\prime \prime}=f(t, x), \\
x(0)=x(1)=0,
\end{array}\right.
$$

where function $f$ satisfies the following conditions:

(A1) $f$ and $f_{x}$ are $C(I \times R)$-functions;

(A2) $f(t, 0) \equiv 0$;

(B1) $f(t, x)>c|x|^{p}$ for $t \in I, x<-M$, where $c>0, p>1, M>0$ are constants;

(B2) $f(t, x) \geq 0$ for $t \in I, x \geq M$;

(A6) solutions of equation (5.1) can extend to the interval $(0 ; 1]$.

Theorem 2. Let the conditions $(A 1),(A 2),(B 1),(B 2)$ and $(A 6)$ hold. Suppose that a solution $y(t)$ of the Cauchy problem

$$
\left\{\begin{array}{l}
y^{\prime \prime}=f_{x}(t, 0) y \\
y(0)=0, \quad y^{\prime}(0)=1
\end{array}\right.
$$

has exactly $n \geq 1$ zeros in the interval $(0,1)$ and $y(1) \neq 0$. Then problem (5.1) has at least $(2 n-1)$ solutions.

Lemma 3. There exists a solution $\xi(t)$ of problem (5.1), $\xi(0)=0, \xi^{\prime}(0)<0$ such that $\xi(t)$ has exactly one zero in the interval $(0 ; 1]$.

This can be proved, using conditions (B1), (B2) and the technique of Section 4 of the work [1].

Now we can prove Theorem 2.

Proof. First we note that there exists a solution $\eta(t)$ of problem $(5.1), \eta(0)=$ $0, \eta^{\prime}(0)>0$ such that $\eta(t)$ does not vanish in the interval $(0 ; 1]$. This follows from conditions (B2) and (A6).

Let $\xi(t)$ be a solution which is described in Lemma 3. Behaviuors of $\eta(t)$ and $\xi(t)$ are shown in Fig. 4. The rest of the proof is similar to that of Theorem 


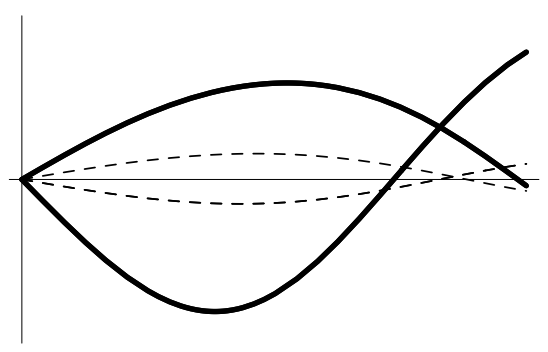

Figure 4. Visualization of Theorem 2.

1. Consider solutions $x(t ; \gamma)$ of equation (5.1), which satisfy initial conditions (2.4). We use the polar coordinates given by formulas (3.3). Consider the functions $\varphi(t ; \gamma)$ and $\rho(t ; \gamma)$ which satisfy initial conditions (3.4).

The linear equation of variations (5.2), converted into polar coordinates using the formulas

$$
y=r \sin \Theta, \quad y^{\prime}=r \cos \Theta
$$

is given by

$$
\left\{\begin{array}{l}
\Theta^{\prime}=\cos ^{2} \Theta-f_{x}(t, 0) \sin ^{2} \Theta, \\
r^{\prime}=r \sin \Theta \cos \Theta+f_{x}(t, 0) r \sin \Theta \cos \Theta .
\end{array}\right.
$$

Since a solution $y(t)$ of problem (5.2) has exactly $n$ zeros in $(0,1)$ and $y(1) \neq 0$ one has that $\pi n<\Theta(1)<\pi(n+1)$. It follows from the proof of Theorem 15.11 in [2] that the same inequalities hold for the angular function $\varphi$, that is,

$$
\pi n<\varphi(1 ; \gamma)<\pi(n+1),
$$

if $\gamma>0$ is small enough. On the other hand

$$
0<\varphi\left(1 ; \gamma_{\eta}\right)<\pi
$$

where $\gamma_{\eta}:=\eta^{\prime}(0)$. Comparison of (5.3) and (5.4) gives that there exist at least $n$ solutions for $\gamma \in\left(0, \gamma_{\eta}\right)$.

Consider the functions $\varphi(t ; \gamma)$ and $\rho(t ; \gamma)$ which satisfy the initial conditions

$$
\varphi(0 ; \gamma)=\pi, \quad \rho(0 ; \gamma)=|\gamma| .
$$

The linear equation of variations (5.2) turns to

$$
\left\{\begin{array}{l}
\Theta^{\prime}=\cos ^{2} \Theta-f_{x}(t, 0) \sin ^{2} \Theta, \\
r^{\prime}=r \sin \Theta \cos \Theta+f_{x}(t, 0) r \sin \Theta \cos \Theta .
\end{array}\right.
$$

Consider $\Theta(t)$, defined by the initial condition $\Theta(0)=\pi$. Since a solution $y(t)$ of equation (5.2), which satisfies initial conditions $y(0)=0, y^{\prime}(0)=-1$, has exactly $n$ zeros in $(0,1)$ and $y(1) \neq 0$, one has that $\pi(n+1)<\Theta(1)<\pi(n+2)$. The same inequalities hold for the angular function $\varphi$, that is, 


$$
\pi(n+1)<\varphi(1 ; \gamma)<\pi(n+2),
$$

if $\gamma<0$ is small enough. On the other hand

$$
2 \pi<\varphi\left(1 ; \gamma_{\xi}\right)<3 \pi
$$

where $\gamma_{\xi}:=\xi^{\prime}(0)$. Comparison of (5.6) and (5.7) gives that there exist at least $\frac{\pi(n+1)-2 \pi}{\pi}=(n-1)$ solutions for $\gamma \in\left(\gamma_{\xi}, 0\right)$. Totally the problem has at least $n+n-1=2 n-1$ nontrivial solutions.

Example 1. Consider the problem

$$
\left\{\begin{array}{l}
x^{\prime \prime}=-40\left(t^{2}+1\right) x+(t+1) x^{2}, \\
x(0)=x(1)=0 .
\end{array}\right.
$$

Figure 5 shows solutions of the equation satisfying the initial conditions $x(0)=0, x^{\prime}(0)=\gamma$, where $\gamma$ is small and positive. This solution has 2 zeros. Figures 5 a) b) show that zeros move to the right as $\gamma$ increases. Figures 5 c) show that the second zero has escaped the interval while the first zero still belongs to the interval. Figure $5 \mathrm{~d}$ ) shows that all zeros have escaped the interval. Therefore there exist two solutions of the given BVP if $\gamma>0$ changes from zero to large enough values.

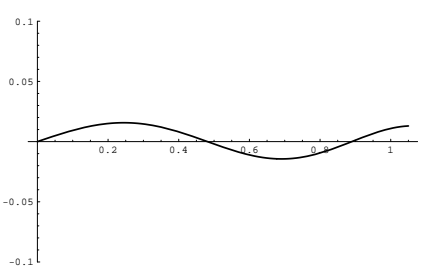

a)

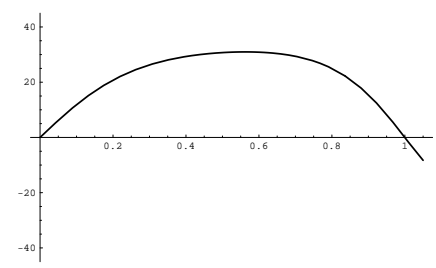

c)

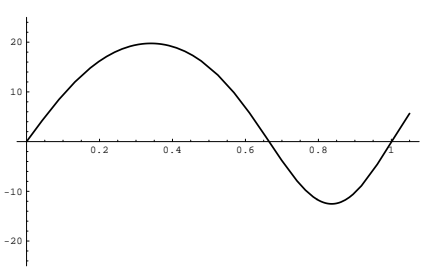

b)

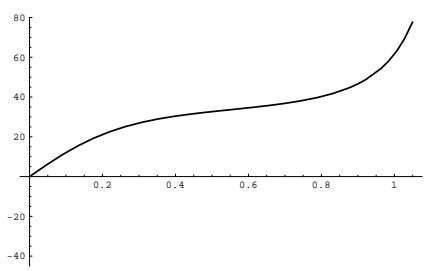

d)

Figure 5. a) $x^{\prime}(0)=0.1 \quad$ b) $x^{\prime}(0)=100 \quad$ c) $x^{\prime}(0)=125.65 \quad$ d) $x^{\prime}(0)=128$.

Figure 6 shows solutions of the equation with $x(0)=0, x^{\prime}(0)=\gamma<0$, for $\gamma$ small enough. Parts a) and b) of the figure show that the zeros move to the right if $\gamma$ decreases. Figures $6 \mathrm{c}$ ), d) show that all zeros except one 


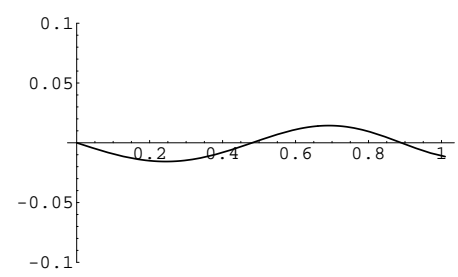

a)

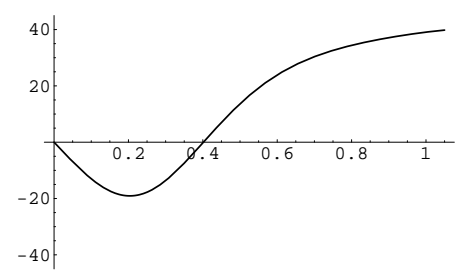

c)

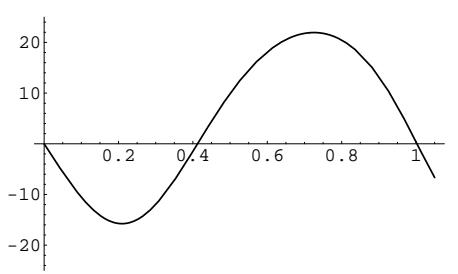

b)

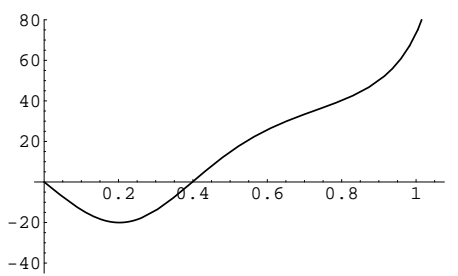

d)

Figure 6. a) $x^{\prime}(0)=-0.1 \quad$ b) $x^{\prime}(0)=-114 \quad$ c) $x^{\prime}(0)=-141 \quad$ d) $x^{\prime}(0)=-149$.

have escaped the interval. Therefore there exists one solution of the given BVP if $\gamma<0$ changes from zero to $-\infty$. Then problem (5.8) has 3 nontrivial solutions.

\section{Acknowledgment}

This work is supported by the project

VPD1/ESF/PIAA/04/NP/3.2.3.1/0003/0065.

\section{References}

[1] Yu.A. Klokov and F.Zh. Sadyrbaev. On the number of solutions of the second order boundary value problems with nonlinear asymptotics. Differential equations, 31(4), 471-479, 1998. (Russian)

[2] M.A. Krasnoselskii and et al. Planar vector fields. Fizmatgiz, Moscow, 1963 (Russian). English transl.: Acad. Press, New York, 1966

[3] L.A. Peletier and W.C. Troy. Spatial Patterns: Higher Order Models in Physics and Mechanics. Birkhauser, Boston, 2001.

[4] A.I. Perov. On a two-point boundary value problem. DAN SSSR, 122(6), 982985, 1958. (Russian)

[5] A.I. Perov. On a boundary value problem for a system of two differential equations. DAN SSSR, 144(3), 1962. (Russian)

[6] V. Radulescu. Finitely many solutions for a class of boundary value problems with superlinear convex nonlinearity. Archiv der Mathematik, 84, 538-550, 2005 . 
[7] B. Ruf and P.N. Srikanth. Multiplicity results for ODE's with nonlinearities crossing all but a finite number of eigenvalues. Nonlinear Analysis:TMA, 10(2), 157-163, 1986.

[8] R. Seydel. Practical Bifurcation and Stability Analysis. Springer Verlag, New York, 1994. Reprint in China: Beijing World Publishing Corporation, 1999

[9] F. Tricomi. Differential equations. Blackie \& Son Ltd, 1961. (Russian transl., Moscow, 1962) 